\title{
The age of the Galaxy's thick disk
}

\author{
Sofia Feltzing ${ }^{1} \dagger$ and Thomas Bensby ${ }^{2}$ \\ ${ }^{1}$ Lund Observatory, \\ Box 43, SE-22100, Lund, Sweden \\ email: sofia@astro.lu.se \\ ${ }^{2}$ European Southern Observatory, \\ Alonso de Cordova 3107, Vitacura, Casilla 19001, Santiago, Chile \\ email: tbensby@eso.org
}

\begin{abstract}
We discuss the age of the stellar disks in the solar neighborhood. After reviewing the various methods for age dating, we discuss current estimates of the ages of both the thinand the thick disks. We present preliminary results for kinematically-selected stars that belong to the thin- as well as the thick disk. All of these dwarf and sub-giant stars have been studied spectroscopically and we have derived both elemental abundances as well as ages for them. A general conclusion is that in the solar neighborhood, on average, the thick disk is older than the thin disk. However, we caution that the exclusion of stars with effective temperatures around $6500 \mathrm{~K}$ might result in a biased view of the full age distribution for the stars in the thick disk.
\end{abstract}

Keywords. Galaxy: structure, Galaxy: disk, kinematics and dynamics, solar neighborhood, Hertzsprung-Russell diagram, stars: late-type

\section{Introduction}

The age of a stellar population can be determined in several ways. For groups of stars, isochrones may be fitted to the stellar sequence in the Hertzsprung-Russell diagram (HRD; see, e.g., Schuster et al. 2006), or the luminosity function for the white dwarfs can be fitted with cooling tracks (e.g., Leggett et al. 1998). Ages for individual stars can be determined from the HRD (if the star is a turn-off or sub-giant star) or by utilizing relations that relate the rotation or atmospheric activity of a star to its age (examples are given by Barnes 2007 and Mamajek \& Hillenbrand 2008). Asteroseismology provides the possibility to constrain the stellar ages very finely. A recent example of the age determination for a young star is given in Vauclair et al. (2008). Finally, the age of a star can be estimated by studying the amount of various elements present in the photosphere of the star. In particular the amount of elements such as $U$ and Th that decay radioactively can be used to estimate the age. Examples of this are given by del Peloso et al. (2005). Estimating the age from the decay of radioactive isotopes is sometimes called nucleocosmochronology.

All but one of these methods, nucleocosmochronology, relies on our understanding of stellar evolution. Some of the methods work well for young stars. This is especially true for rotation and stellar activity (see Mamajek 2009 and Barnes 2009) while the determination of stellar ages using isochrones is limited in various ways depending on the type of star under study.

The isochrones give the best results for turn-off and sub-giant stars, but with very poor power to differentiate between different ages on the red giant branch. In fact, the stars on the sub-giant branch are the most desirable tracers of the age of a particular

$\dagger$ SF is a Royal Swedish Academy of Sciences Research Fellow supported by a grant from the Knut and Alice Wallenberg Foundation. 
stellar population (Sandage et al. 2003). In particular, it does not matter if the stellar temperature is well determined or not (Bernkopf \& Fuhrmann 2006).

However, we would argue that the power of isochrone ages mainly lies in the relative ages - i.e., being able to say "star A is older than star B and it is about this big an age difference between star A and star B." Such statements and determinations are, of course, less desirable if we want to determine the absolute age of a star or stellar population, but they are very powerful if we want to know in which order the stars formed and what time-scales were involved, i.e., the study of galaxy formation and evolution. The good thing with the isochrone method is that it is reasonably straightforward to derive the ages also for large samples of stars (but see Jørgensen \& Lindegren 2005) as well as for old stars. The less useful aspect is that we are mainly limited to using the turn-off stars. For an older population this implies the inherently faint, but numerous, F- and G-type dwarf and sub-giant stars. In order to construct the HRD, we need to know the distances to the stars. This is difficult to do for large numbers of stars once we are outside the volume covered by Hipparcos. However, it is possible to derive the distance if the star is assumed to be a dwarf or if the star can be determined to be a dwarf star. Strömgren photometry and some other photometric systems are able to determine the evolutionary state of a star. Some examples of how the Strömgren photometry can be used to this end are given in Schuster et al. (2006), von Hippel \& Bothun (1993), and Jønch-Sørensen (1995). So far these studies have mainly been limited to the solar neighbourhood due to the observational equipment available. Recent studies are trying to remedy this situation by using CCD images obtained with wide-field cameras. An early example is given in Árnadottír et al. (2008).

\section{The ages of the stellar disks}

The main tracers for age-dating the thin disk are open clusters, the luminosity function of white dwarfs, and, recently, nucleocosmochronology. Generally, estimates of the age of the thin disk using the luminosity function of white dwarfs find a lower limit for the age of around 9 Gyr (e.g Oswalt et al. 1995; Leggett et al. 1998; Knox et al. 1999).

Open clusters indicate a similar lower age for the thin disk. It is interesting to note the existence of open clusters that are both old as well as metal-rich. NGC 6791 has a metallicity of +0.35 dex and an age between 8 and 9 Gyr (Grundahl et al. 2008). Such old stars are normally not considered to be able to be that metal-rich. In our new, local sample of stars we seem to pick up a few metal-rich and old stars that have thin disk kinematics and thin disk abundance patterns.

The thin disk hosts the majority of the younger stars. In general young stars rotate more rapidly than older stars and they have more chromospheric activity. As they grow older they rotate more slowly and their outer atmospheres become less active. These characteristics can be utilized to estimate the age of a star (Mamajek \& Hillenbrand 2008; Barnes 2007). However, none of these measures are particularly straightforward. A recent example of how they could be combined in order to give better age estimates is given by Mamajek \& Hillenbrand (2008). They show that with their new measure of stellar ages, combined from rotation and activity measures, the star formation history of the thin disk has been less variable than previously thought.

The thick disk is in general found to be exclusively old. The age estimates for the thick disk have been done either by studying local, kinematically-selected samples, or by studying the turn-off color for stars well above the Galactic plane where the thick disk dominates (typically about $1 \mathrm{kpc}$ and higher; Gilmore et al. 1995). 
Recent studies of kinematically-selected thick disk samples in the solar neighborhood appear to agree that the thick disk is old and, essentially, all older than kinematicallydefined thin disk samples (see, e.g., Bensby et al. 2005; Reddy et al. 2006). It is clear that the kinematic definitions are only statistical and that we will never be able to create a sample that is completely free from thin-disk stars. It is especially important to keep in mind that the young stars in the stellar disk (thin or thick) have a rather lumpy distribution in velocity space. This enables the identification of stars that potentially have a common origin but it complicates the division of stars into thin- and thick disk (for a recent discussion see Holmberg et al. (2007)). As shown in Holmberg et al. (2007), as we progress to older stars the kinematics change and the velocity distributions get smoother. This should not be surprising as any older sample will be more dominated by the thick disk, for which not much lumpiness has been observed so far (but see Gilmore et al. (2002), Schuster et al. (2006), and Wyse et al. (2006) for discussions of the last merger and how that has influenced the local as well as not-so-local stellar kinematics). Not only the velocity dispersions are important to consider but also how large a portion the thick disk contributes in the solar neighborhood (the normalization of the stellar number density). In Bensby et al. (2005), we show that our selection criteria are rather robust against changes in this normalization. It remains to be fully investigated how sensitive the selection criteria are to the presence of lumpy velocity distributions.

In this context the study of volume-limited samples becomes increasingly important. Fuhrmann (2008) studied a volume-limited sample of stars within 25 pc from the Sun. He identifies the stars that are enhanced in $[\mathrm{Mg} / \mathrm{Fe}]$ with the thick disk. All of these stars are found to be older than the stars he associates with the thin disk, but no specific ages are given. In the next section we will revisit the volume-limited samples in comparison to the samples selected based on kinematics.

\section{A new local sample of late-F and early-G dwarf stars - the local $\operatorname{disk}(\mathrm{s})$ revisited}

We have obtained high-resolution, high $\mathrm{S} / \mathrm{N}$ spectra for about 900 dwarf stars. The data have been obtained with several spectrographs but in general $\mathrm{S} / \mathrm{N}>250$ and $R \geqslant$ 65,000 (apart from the subset of stars originally observed with FEROS [Bensby et al. (2003)] which have $R=48,000)$. In Feltzing \& Bensby (2008) we presented the kinematic properties and some elemental abundances for a subsample of about $550 \mathrm{~F}$ - and $\mathrm{G}$ dwarf stars.

The ages for these stars have been derived using Yonsei-Yale isochrones (see Bensby et al. 2005), where we also allow for enhancement in $\alpha$-elements. Taking the $\alpha$-enhancement into account is important because for a given star the age will be lower should it be enhanced in these elements as opposed to if it is not. The effect of taking the $\alpha$-enhancement into account is thus that any age-gap between the thick- and the thin disk decreases (the thin disk stars are not at all or only moderately enhanced in $\alpha$-elements and thus there is only a small or no effect on their ages when $\alpha$-enhancement is included in the age estimates).

In Fig. 1a and Fig. 1c we are attempting a comparison with results from Fuhrmann (2008) and show the stars within 25 and 50 pc, respectively. In the plots, we have also coded the age of the stars such that an older star has a bigger symbol. It is clear from Fig. 1a and Fig. 1c that stars that are enhanced in an $\alpha$-element, here Si, are also older than stars that are not enhanced. On average, our volume-limited samples appear to show the same sort of trends that Fuhrmann (2008) found. Although the sample within 
$25 \mathrm{pc}$ is very small and incomplete, still stars enhanced in Si are old and the young stars are not enhanced and also show a tight trend of $[\mathrm{Si} / \mathrm{Fe}]$ vs. $[\mathrm{Fe} / \mathrm{H}]$. Fig. 1c further shows that there appears to be a real separation between the two trends, i.e., one for younger and one for older stars. It should be kept in mind that our sample is not volume-complete and also that Fuhrmann (2008) imposes some further criteria on the stellar parameters for those stars that he includes in his final plots. For now, we are showing all stars, covering the full parameter space sampled within our program (compare, e.g., Feltzing \& Bensby (2008) and Bensby et al. (2009, in preparation)).

Figures $1 \mathrm{~b}$ and $\mathrm{d}$ then show the volume-limited samples but with a kinematic selection imposed as well such that we select stars that are ten times more likely to be thick disk than thin disk to represent the thick disk and vice versa for the thin disk. It is intriguing to see that the kinematically-selected thin disk stars mimic the trend found for the younger stars and the thick disk mimics the trend found for the older stars.



Figure 1. $[\mathrm{Si} / \mathrm{Fe}]$ vs. $[\mathrm{Fe} / \mathrm{H}]$ for four subsamples drawn from the full sample of Bensby et al. (2009, in preparation). All stars shown have age determinations with relative errors less than 2 Gyr. Note that these are not volume-complete samples, only volume-limited. a. All stars from Bensby et al. within $25 \mathrm{pc}$. The size of the symbols indicate their ages with larger symbols representing higher ages. The scale for the ages is indicated at the lower part of the panel. b. A kinematically-selected subset of the stars in $\mathbf{a}$. A - marks stars that are ten times more likely to be thick- than thin disk members and a $\circ$ marks stars that are ten times more likely to be thinthan thick disk members. c. All stars from Bensby et al. within 50 pc. The size of the symbols indicate their ages with larger symbols representing higher ages. Same sizes are used as in panel a. d. A kinematically-selected subset of the stars in c. A - marks stars that are ten times more likely to be thick- than thin disk members and a $\circ$ marks stars that are ten times more likely to be thin- than thick disk members. Stars marked with an additional $\times$ are stars that are ten times more likely to be thin disk members than thick disk but also have an age larger than 8 Gyr. 
In Feltzing \& Bensby (2008) we identified a small number of stars on typical thin-disk orbits but with enhanced abundances for the $\alpha$-elements. These stars were found to be old (older than about 8 Gyr in our determination). In Fig. 1d these stars are explicitly marked. For further discussion about plausible origins for these stars we refer to Feltzing \& Bensby (2008). It is worth noting, however, that it is essentially these stars that make the downward trend of $[\mathrm{Si} / \mathrm{Fe}]$ in the kinematically-selected thick disk sample blend in with the thin-disk sample.

Figure 2 shows the age-metallicity plot for a first selection of stars with kinematics that make them very likely thick-disk candidates. All of these stars are ten times more likely to belong to the thick- than to the thin disk. As can be seen, the bulk of these stars are older than the Sun and they have a mean age of around 10 Gyr. They cover that whole metallicity range from -1 dex to solar. For this first attempt at establishing if there is an age-metallicity relation present in our kinematically defined sample, we have only included stars for which we could determine the ages to better than 2 Gyr. As our stars originally are essentially selected only based on their kinematic properties and a metallicity estimated from photometry, we cover a reasonably large range of $\mathrm{T}_{\text {eff }}$. In Fig. 2 we have chosen to show the stars with $\mathrm{T}_{\text {eff }}>6000 \mathrm{~K}$ with a separate symbol. Not surprisingly, these stars are in general young. If they really belong to the thick disk then that would be rather challenging for any of the models put forward for the formation of the thick disk. However, our method to determine the stellar ages is "simple" and as these apparently young stars are in regions of the HRD where the stellar tracks show various "kinks" such a simple age estimate might go wrong in the estimate of the error. We will therefore redo all our ages using the method developed by Jørgensen \& Lindegren (2005). This method provides a better and more realistic estimate of the error in the age determination. For now we would, however, like to caution against over-interpreting apparent young ages present in kinematically-defined thick-disk samples.

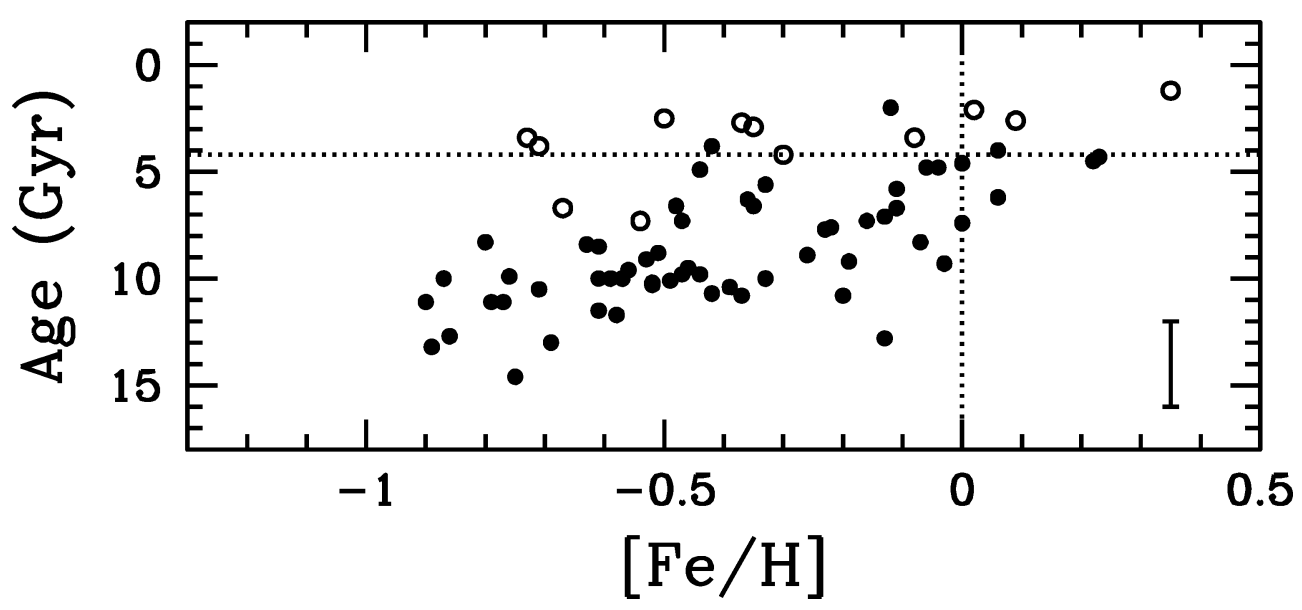

Figure 2. Ages and metallicities for a sample of thick disk stars from our new study. The stars shown all are ten times more likely to belong to the thick as opposed to the thin disk. The estimated errors in the derived ages are less than 2 Gyr. The error-bar in the lower right hand corner shows a 2 Gyr error. Metallicities are based on spectroscopy. The position of the Sun is marked by two dotted lines. $\alpha$-enhancement has been taken into account when determining the ages (see Sect. 3). The filled circles show stars with $\mathrm{T}_{\text {eff }}<6000 \mathrm{~K}$ and the open circles the 9 thick disk stars that have $\mathrm{T}_{\text {eff }}>6000 \mathrm{~K}$. 


\section{Summary}

Age determinations of the stellar disk(s) of the Galaxy are inherently complicated. There are several factors that make it hard to define the age of either disk, not least the mixture of stellar populations in the solar neighborhood. The absolute ages of individual stars may be obtained through, e.g., asteroseismology and nucleocosmochronology. However, for the study of the stellar populations as such, fitting of isochrones to well-defined samples and the fitting of the white dwarf luminosity function using cooling tracks might be more appropriate.

In most current studies, stars with kinematics typical of the thick disk are, on average, found to be older than stars with kinematics typical of the thin disk. There appears to be an age-metallicity relation present in the thick disk. This is found in studies using various techniques. However, the exact definition of the thick disk in relation to the thin disk in terms of stellar kinematics is not straightforward and will need more work.

\section{References}

Árnadottír, A. S., Feltzing, S., \& Lundström, I. 2008, ArXiv:0807.1665

Barnes, S. A. 2009, these proceedings

Barnes, S. A. 2007, ApJ, 669, 1167

Bensby T., Feltzing, S., \& Lundström, I. 2003, $A \mathscr{E} A$, 410, 527

Bensby T., Feltzing, S., Lundström, I., \& Ilyin, I. 2005, A\&A A, 433, 185

Bernkopf, J. \& Fuhrmann, K. 2006, MNRAS, 369, 673

del Peloso, E. F., da Silva, L., Porto de Mello, G. F., \& Arany-prado, L. I. 2005, A\& A, 440, 1153

Feltzing, S. \& Bensby, T. 2008, arXiv: 0811.1777

Fuhrmann, K. 2008, MNRAS, 384, 173

Gilmore, G., Wyse, R. F. G., \& Jones, J. B. 1995, AJ, 109, 1095

Gilmore, G., Wyse, R. F. G., \& Norris, J. E. 2002, ApJ, 574, L39

Grundahl, F., Clausen, J. V., Hardis, S., \& Frandsen, S. 2008, arXiv:0810.2407G

Holmberg, J., Nordström, B., \& Andersen, J. 2007, A\&SA, 475, 519

Jønch-Sørensen, H. 1995, A\&A, 298, 799

Jørgensen, B. R. \& Lindegren, L. 2005, A\&A, 436, 127

Knox, R. A., Hawkins, M. R. S., \& Hambly, N. C. 1999, MNRAS, 306, 736

Leggett, S. K., Ruiz, M. T., \& Bergeron, P. 1998, ApJ, 497, 294

Mamajek, E. E. 2009, these proceedings

Mamajek, E. E. \& Hillenbrand, L. A. 2008, ApJ, 687, 1264

Oswalt, T. D., Smith, J. A., Wood, M. A., \& Hintzem, P. 1995, Nature, 382, 692

Reddy, B. E., Lambert, D. L., \& Allende Prieto, C. 2006, MNRAS, 367, 1329

Sandage, A., Lubin, L. M., \& VandenBerg, D. A. 2003, PASP, 115, 1187

Schuster, W. J., Moitinho, A., Mrquez, A., Parrao, L., \& Covarrubias, E. 2006, A\&SA, 445, 939

Vauclair, S., Laymand, M., Bouchy, F., Vauclair, G., Bon Hoa, A. H., Charpinet, S., \& Bazot, M. 2008, A\&A A 482, L5

von Hippel, T. \& Bothun, G. D. 1993, ApJ, 407, 115

Wyse, R. F. G., Gilmore, G., Norris, J. E., Wilkinson, M. I., Kleyna, J. T., Koch, A., Evans, N. W., \& Grebel, E. 2006, ApJ, 639, L13

\section{Discussion}

R. WYSE: The elemental abundance pattern (enhanced $[\alpha / \mathrm{Fe}]$, type II dominated) of the thick disk implies a short duration, less than the time for iron from Type Ia, $\sim 1$ Gyr. How is this consistent with a 5 Gyr or so spread in ages? Also the thin-disk kinematics are not well modeled by Gaussians so I would caution against population assignment based on Gaussians. 
S. Feltzing: I agree that at least the thin disk is not well modeled by a Gaussian. It is obvious that the distribution of the velocities for nearby F- and G dwarf stars is lumpy. About abundances and ages: Taking a second look at our sample and also looking at the enhancement of $\alpha$-elements, in this case $\mathrm{Ti}$, I would say that the data might still be compatible with the picture you sketch in that the majority of the thick disk stars are enhanced in $\alpha$ elements, and their mean age is $\sim 10$ Gyr with some scatter, and there is a younger group with $\alpha$ about solar.

A. FREBEL: What are the abundance trends for stars in the $z$-selected boxes? Similar trends; different trends?

S. Feltzing: The abundance trends for our sample for the different boxes differ. For example, the box with high eccentricity and high $z_{\max }$ shows a very tight trend, e.g., for $\mathrm{Ti}$, while stars with low eccentricity and high $z_{\max }$ show a much less tight trend and also potentially different levels of $\alpha$ enhancement.

J. Melbourne: I was interested in the younger stars identified in the thick disk. Does this argue for a more extended process of thick disk formation than a single monolithic event?

S. Feltzing: When a kinematic decomposition of the stars into a thin- and thick disk is done (e.g., Bensby et al. 2003) there are essentially no stars younger than about solar age. The thin disk stars selected in the same way appear to show a younger mean age than the thick disk stars. I am not sure we are in a position yet to interpret the few apparently young thick disk stars in terms of monolithic collapse or not. I still think a formation of a thickened disk through a merger event is favored by most of the data. This means today's thick disk stars formed in a thin disk.

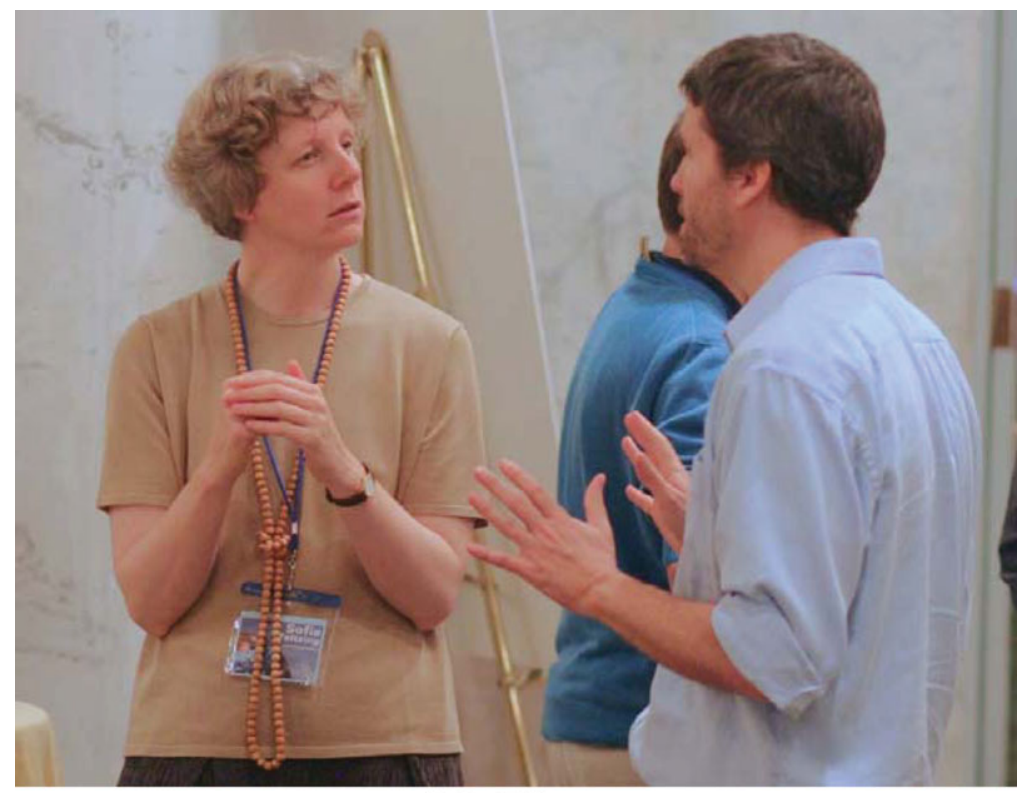

Sofia Feltzing 


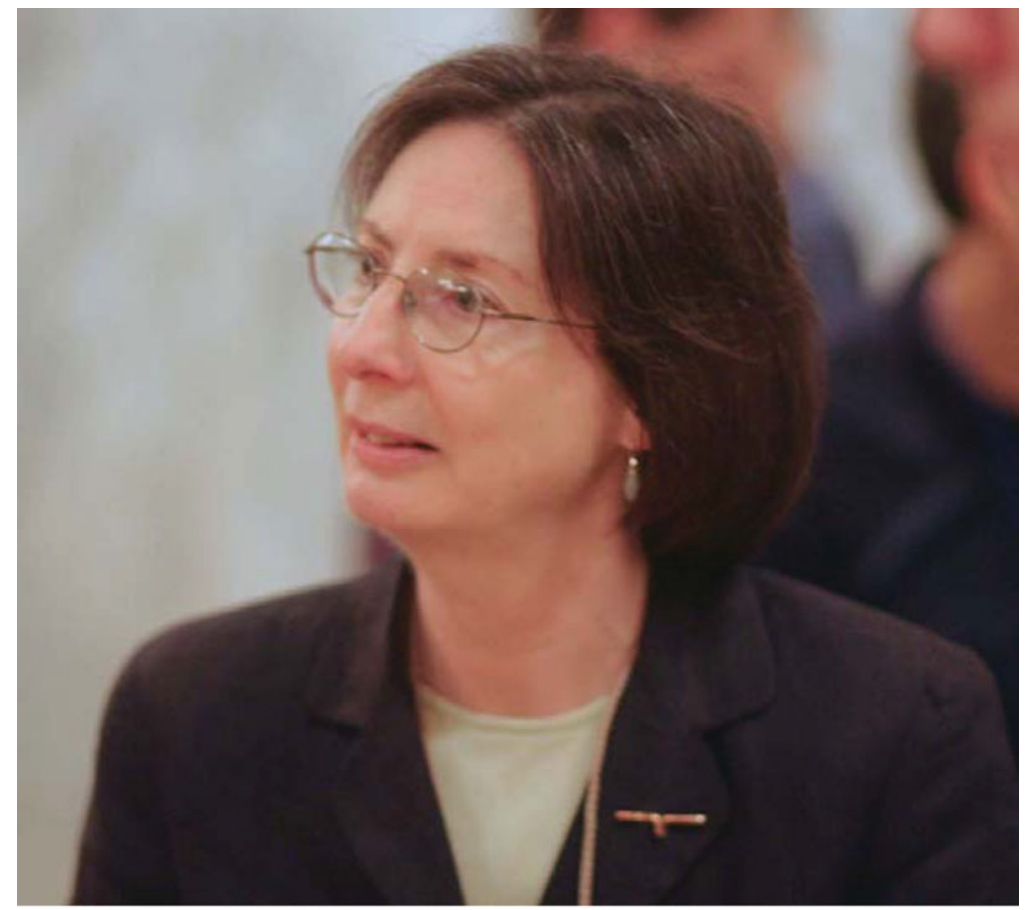

Rosie Wyse

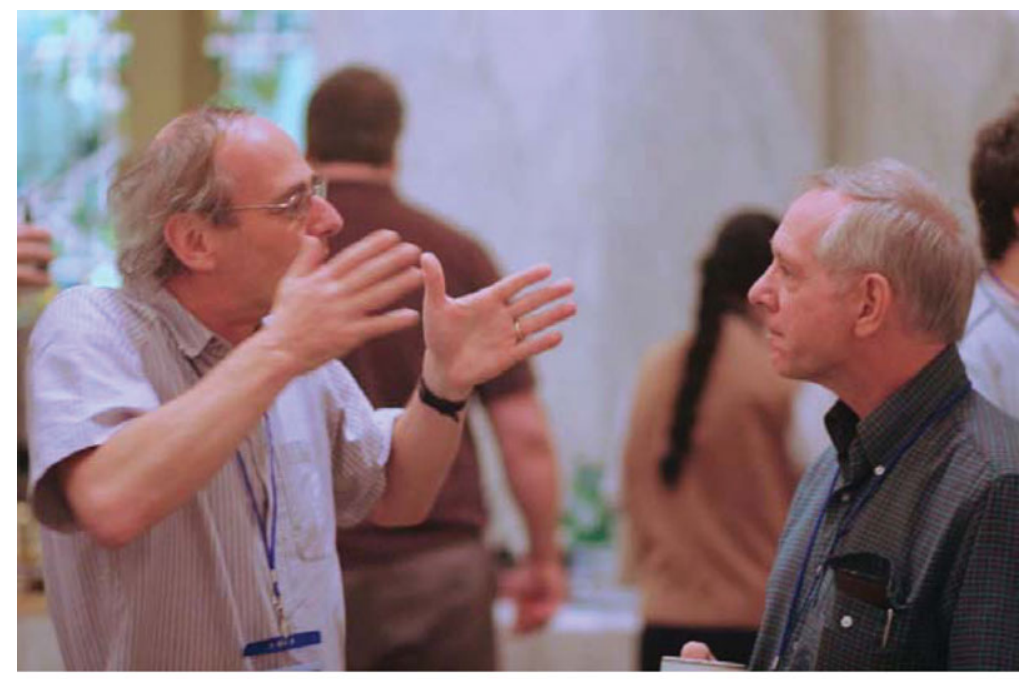

George Meynet and Chris Corbally 